\title{
Venom Alkaloid and Cuticular Hydrocarbon Profiles Are Associated with Social Organization, Queen Fertility Status, and Queen Genotype in the Fire Ant Solenopsis invicta
}

\author{
Dorit Eliyahu • Kenneth G. Ross • Kevin L. Haight • \\ Laurent Keller • Jürgen Liebig
}

Received: 7 January 2010 /Revised: 10 October 2011 /Accepted: 4 November 2011 /Published online: 18 November 2011

(C) Springer Science+Business Media, LLC 2011

\begin{abstract}
Queens in social insect colonies advertise their presence in the colony to: a) attract workers' attention and care; b) gain acceptance by workers as replacement or supplemental reproductives; c) prevent reproductive development in nestmates. We analyzed the chemical content of whole body surface extracts of adult queens of different developmental and reproductive stages, and of adult workers from monogyne (single colony queen) and polygyne (multiple colony queens) forms of the fire ant Solenopsis invicta. We found that the composition of the most abundant components, venom alkaloids, differed between queens and workers, as well as between reproductive and non-reproductive queens. Additionally, workers of the two forms could be distinguished by alkaloid composition. Finally, sexually mature, non-reproductive queens from polygyne colonies differed in their proportions of cis-
\end{abstract}

Electronic supplementary material The online version of this article (doi:10.1007/s10886-011-0037-y) contains supplementary material, which is available to authorized users.

D. Eliyahu $\cdot$ K. L. Haight $\cdot$ J. Liebig $(\bowtie)$

School of Life Sciences, Arizona State University,

Tempe, AZ, USA

e-mail: Juergen.Liebig@asu.edu

K. G. Ross

Department of Entomology, University of Georgia,

Athens, GA, USA

L. Keller

Department of Ecology and Evolution, University of Lausanne,

Lausanne, Switzerland

Present Address:

D. Eliyahu

Department of Entomology, University of Arizona,

Tucson, AZ, USA piperidine alkaloids, depending on their $G p-9$ genotype, although the difference disappeared once they became functional reproductives. Among the unsaturated cuticular hydrocarbons characteristic of queens, there were differences in amounts of alkenes/alkadienes between nonreproductive polygyne queens of different $G p-9$ genotypes, between non-reproductive and reproductive queens, and between polygyne and monogyne reproductive queens, with the amounts increasing at a relatively higher rate through reproductive ontogeny in queens bearing the $G p-9$ $b$ allele. Given that the genotype-specific piperidine differences reflect differences in rates of reproductive maturation between queens, we speculate that these abundant and unique compounds have been co-opted to serve in fertility signaling, while the cuticular hydrocarbons now play a complementary role in regulation of social organization by signaling queen $G p-9$ genotype.

Key Words Cuticular hydrocarbons · Fertility signal . Piperidines · Queen pheromone - Reproductive division of labor Venom alkaloids $\cdot$ Hymenoptera $\cdot$ Formicidae

\section{Introduction}

Communication plays a central role in the organization of ant colonies. It is especially important for queens to communicate their reproductive potential and status to nestmates. As they mature sexually, queens must avoid aggression by nestmate workers and gain acceptance by workers as new reproductives, if the colony is recruiting such individuals. Once making the transition to reproductive status, queens must attract the attention and care of workers (Ortius and Heinze, 1999; Hannonen et al., 2002). 
At the same time, reproductive queens must produce signals that regulate reproductive development and behavior of other colony members (Keller and Nonacs, 1993). Elucidation of the mechanisms that underlie the communication of individual reproductive potential and status is critical for understanding the organization of ant societies.

A key question regarding these mechanisms is what compounds are used to mediate the interactions between queens and workers? Studies suggest that cuticular hydrocarbons play a central role in nestmate discrimination, queen attraction, and regulation of reproduction in many ants, bees, wasps, and termites (Monnin, 2006; Le Conte and Hefetz, 2008; Liebig et al., 2009; Peeters and Liebig, 2009; Weil et al., 2009; van Zweden and D'Ettorre, 2010). The hydrocarbon characteristic of reproductive queens in the ant Pachycondyla inversa, for example, elicits a strong antennal response in electro-antennograms of workers, thus suggesting a sensory bias to this compound (D'Ettorre et al., 2004). In the ant Aphaenogaster cockerelli, workers with highly active ovaries produce cuticular hydrocarbons identical to those of reproductive queens (Smith et al., 2008); such workers are attacked by nestmates, presumably preventing them from reproducing. These attacks can be triggered in laboratory assays by the alkane $n$-pentacosane, the main component of the hydrocarbon profile of reproductive queens (Smith et al., 2008). Other direct evidence for an important role for hydrocarbon profiles in the regulation of ant reproduction comes from egg discrimination experiments in Camponotus floridanus. Reproductive queens produce a hydrocarbon profile that contains roughly twice the number of components as the profiles of non-reproductive queens (Endler et al., 2006). These reproductive queen hydrocarbon profiles also are present on queen-laid eggs, but not on worker-laid eggs, allowing workers to recognize and eliminate the latter (Endler et al., 2004). Finally, a primer effect of a major hydrocarbon compound produced by fertile queens of the ant Lasius niger has been demonstrated through reduced ovarian development of workers exposed to this compound compared to controls (Holman et al., 2010).

Given the widespread association between cuticular hydrocarbon profiles and reproductive status in eusocial insects, and the direct evidence for these compounds as fertility signals, it is especially interesting to find and explain possible exceptions to this pattern. In the red imported fire ant, Solenopsis invicta, the role of cuticular hydrocarbons has been studied only in the context of nestmate discrimination, with experimental transfer of hydrocarbons having no effect, thus suggesting that these compounds are not involved in this behavior (Anderson and Vander-Meer, 2001). Because cuticular hydrocarbons are involved in nestmate discrimination in many other social insect species (van Zweden and D’Ettorre, 2010), their lack of use in nestmate discrimination for S. invicta suggests that they also may not be involved in fertility signaling. In fact, hydrocarbon patterns for the postpharyngeal glands of queens did not change over the course of 10 days following mating and initiation of oogenesis (Vander Meer et al., 1982), a period during which such signaling to potential reproductive rivals or worker nestmates should be important.

Previous studies have shown that fully reproductive $S$. invicta queens produce a pheromone that inhibits winged, virgin queens from shedding wings (dealating) and developing ovaries (Fletcher and Blum, 1981; Vargo, 1998); workers are not affected in this way, because they have rudimentary ovaries and cannot lay eggs. The inhibitory pheromone(s) apparently originates from the venom sac and the postpharyngeal glands (Vargo and Hulsey, 2000), but the components are yet to be identified. The venom sac also has been shown to be the source of large amounts of venom alkaloids (Brand et al., 1972, 1973) and of pyranones. The latter are attractive to workers (Rocca et al., 1983a,b) but do not inhibit the reproductive development of winged queens (Glancey et al., 1984). In workers, the most abundant components of the alkaloids are trans-2-methyl-6-(cis-4'- $n$-tridecenyl) piperidine (trans- $\mathrm{C}_{13: 1}$ ), trans-2-methyl-6- $n$-tridecylpiperidine (trans$\mathrm{C}_{13}$ ), trans-2-methyl-6-(cis-6'-n-pentadecenyl) piperidine (trans- $\mathrm{C}_{15: 1}$ ), and trans-2-methyl-6- $n$-pentadecylpiperidine $\left(\mathrm{C}_{15}\right)$. In alate queens, the venom constituents are different, with the most abundant component being cis-2-methyl-6- $n$ undecylpiperidine ( $\left.c i s-\mathrm{C}_{11}\right)$ (Brand et al., 1973). While workers inject venom directly into other animals for defense and predation, or spray it throughout the nest environment, presumably for protection against microbial pathogens, queens apply it over eggs as they are laid. It has been suggested that this behavior protects eggs from entomopathogenic fungi (Vander-Meer and Morel, 1995; Tschinkel, 2006). However, this behavior also raises the possibility that venom alkaloids on the surface of eggs may play a role in advertising the presence and fertility status of queens, as do hydrocarbons in other social insects.

An added element of complexity in the pheromonal regulation of reproduction and social organization in $S$. invicta comes from the occurrence of two social forms: monogyne, in which colonies contain a single queen, and polygyne, in which colonies contain multiple queens. The two social forms differ in several ways. For example, monogyne colonies are initiated by a single queen who raises her first brood by using body reserves, while polygyne colonies are initiated by budding, a process in which queens and workers leave the colony to initiate a new colony nearby (see Ross and Keller, 1995). Polymorphism in social organization is associated with allelic variation at the gene $G p-9$; in the USA, monogyne colonies contain only inhabitants bearing the $B$ allele, while polygyne colonies also include inhabitants bearing the $b$ 
allele (Gotzek and Ross, 2007). Monogyne workers (all of which are $B B$ homozygotes) accept a single $B B$ replacement reproductive queen if their colony has been queenless for several days, but they do not tolerate queens bearing the $b$ allele. Alternatively, polygyne workers (a mix of individuals with and without the $b$ allele) accept multiple reproductive queens bearing the $b$ allele, but do not tolerate $B B$ queens (Ross and Keller, 1998, 2002). This genotype-specific pattern of aggression or tolerance of queens by workers extends to nestmate non-reproductive queens in polygyne colonies; worker aggression toward young $B B$ queens escalates as these queens approach two weeks of adult age, coincident with attainment of sexual maturity and reproductive competence (Keller and Ross, 1993a, 1999; Brent and Vargo, 2003). Ultimately, all sexually mature $B B$ queens in polygyne colonies are executed or leave the nest on mating flights (Keller and Ross, 1999). Importantly, worker aggression toward $B B$ queens in polygyne colonies is induced by a transferable queen signal on the cuticle (Keller and Ross, 1998). While production of this signal is related to weight and tendency to initiate egg-laying in non-reproductive queens, it is not related to fecundity in reproductive queens (Keller and Ross, 1993a, 1999; Ross and Keller, 1998). This has led to the suggestion that at least two distinct queenproduced pheromones are involved in regulation of colony queen number and identity in $S$. invicta: one communicating Gp-9 genotype and the other communicating actual or potential reproductive status (Keller and Ross, 1999, Gotzek and Ross, 2007).

Here, we compared venom alkaloid and cuticular hydrocarbon profiles of queens of different stages of sexual maturity, reproductive status, and $G p-9$ genotype, as well as workers, originating from monogyne and polygyne $S$. invicta colonies. If cuticular hydrocarbons do not encode any of the above-mentioned types of information, then the abundant piperidines, unique to the venom of this group of ants, may fill this role instead. Our goal was to detect associations between chemical profiles and reproductive state/genotype of different classes of colony members. Such associations may indicate a role for specific compounds as releaser or primer pheromones involved in communicating Gp-9 genotype and/or fertility status to workers, roles essential to the regulation of colony social organization in fire ants.

\section{Methods and Materials}

Insects Large colonies of each social form of Solenopsis invicta were collected and reared in the laboratory under standard conditions (Ross, 1988). A total of 25 polygyne and 23 monogyne colonies were collected from AthensClarke Co. and Oglethorpe Co., Georgia, USA, respectively.
Social organization of the polygyne colonies was determined by finding multiple reproductive (wingless, egg-laying) queens in each. Social organization of the monogyne colonies was determined by finding only a single reproductive queen in each. Social organization was subsequently confirmed for all colonies by genotyping several reproductive queens (polygyne colonies) or 20 adult workers (monogyne colonies) at the gene $G p-9$, using horizontal starch gel electrophoresis coupled with amido black staining (DeHeer et al., 1999); all polygyne reproductive queens possessed the $B b$ genotype, while all monogyne workers possessed the $B B$ genotype, as expected (Gotzek and Ross, 2007). Colonies were fed daily by alternating a high-protein diet (tuna/dog food/peanut butter mix) with a high-carbohydrate diet (assorted vegetables/ granulated sugar mix). These diets were supplemented with crickets and/or mealworms on a twice-weekly basis.

Several hundred adult workers from individual laboratory colonies were collected by placing a glass vial upright in the foraging area of each rearing unit for several minutes, and allowing workers to fall into the vial; vials were placed briefly on dry ice, then stored at $-80^{\circ} \mathrm{C}$ until extraction. Thirteen colonies of each social form were sampled in this way for determination of piperidine alkaloid profiles, while ten colonies of each form were sampled for determination of hydrocarbon profiles (see Table 1 for sample size information).

We collected queens at three different key stages of sexual and reproductive development. First, we collected 2d-old, sexually immature and non-reproductive, queens from polygyne colonies. These were obtained by transferring 1-6 newly emerged (callow) adult queens to small rearing units with several hundred worker brood and adults from the natal colony (see Table 1 for sample sizes). Forty-eight hours after transfer, queens were collected for chemical analysis. Second, we collected 14d-old queens from polygyne colonies. By this age, queens have undergone extensive weight gain and accumulation of energy reserves (fat and glycogen), characteristic of completion of maturation in $S$. invicta (Keller and Ross, 1993a, 1999; Brent and Vargo, 2003). Such queens are sexually mature, but typically embark on a mating flight and are inseminated before becoming reproductively active. Two to six newly emerged queens from each polygyne source colony were held individually in small rearing units, with worker brood and adults from the natal colony, for $14 \mathrm{~d}$ before being collected for analysis (see Table 1 for sample sizes). These queens were held individually, rather than in groups, because workers execute queens with a $G p-9 B B$ genotype when they attain sexual maturity in colonies that also contain queens with a $B b$ genotype (see Keller and Ross, 1998, 1999). This procedure also ensured that the reproductive development of young queens was not inhibited by the presence of other queens in the same unit. In the rearing 


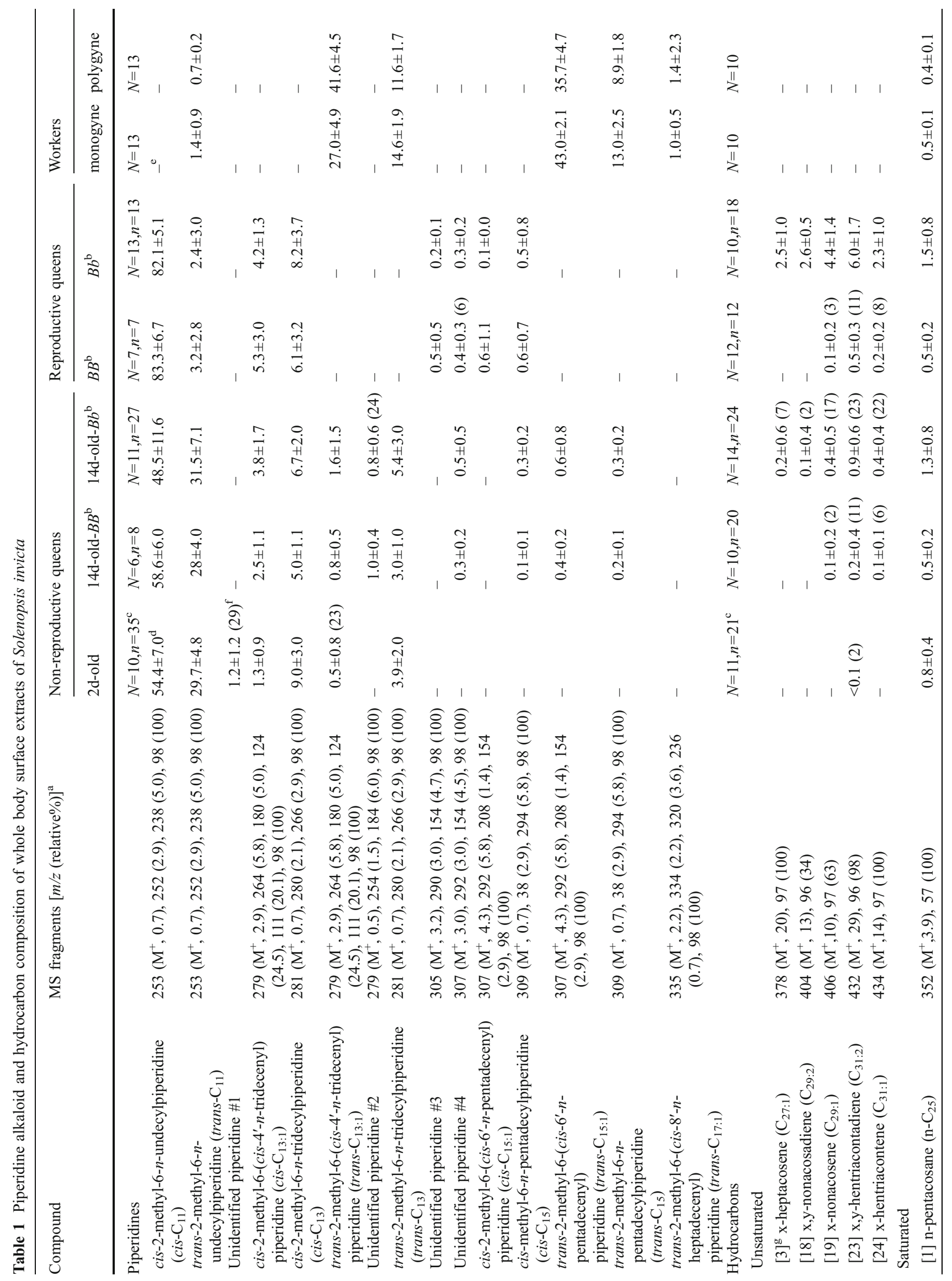




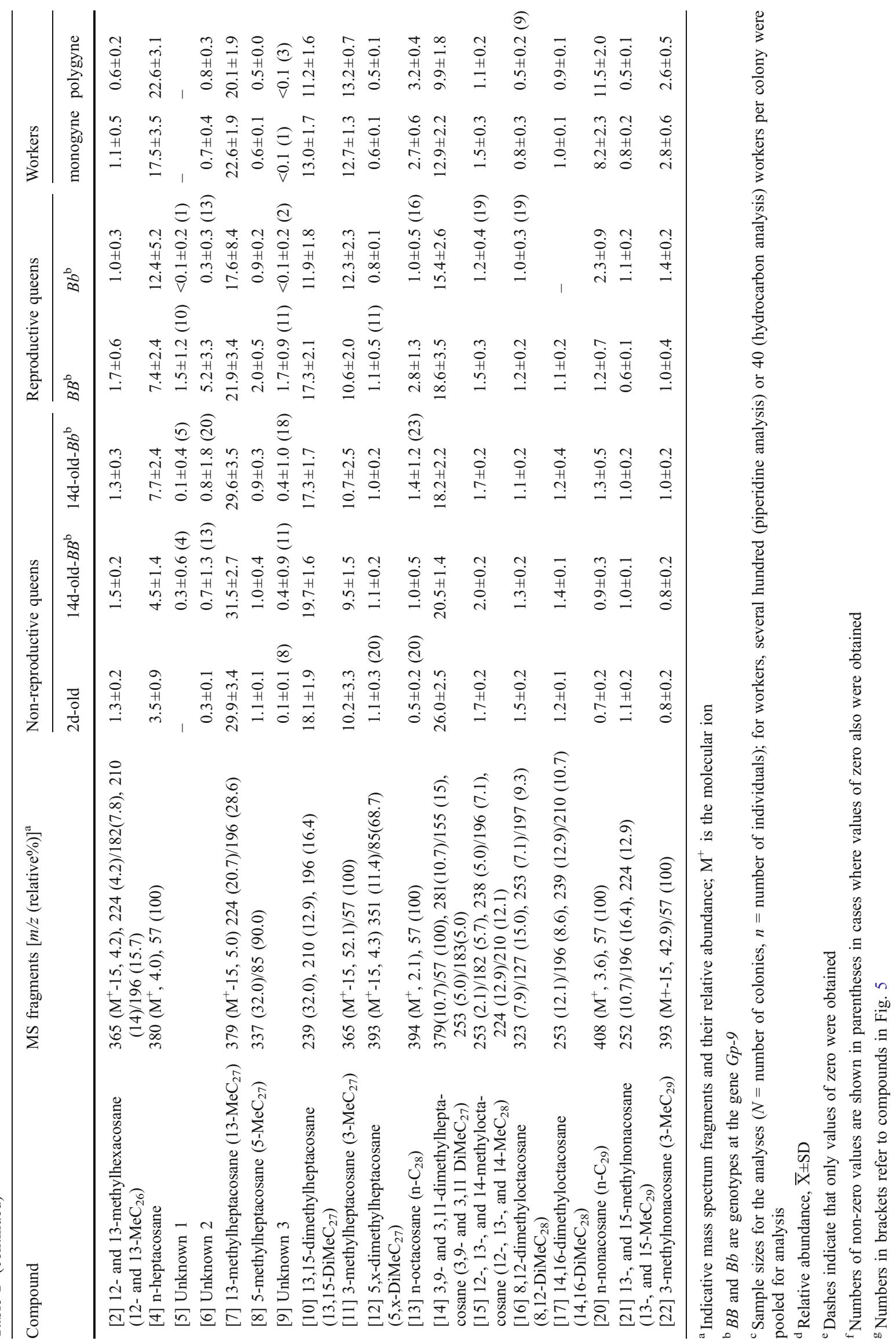


units, some of the 14d-old queens dealated, a behavior associated with onset of oogenesis (Fletcher and Blum, 1981; Brent and Vargo, 2003); the identities of these queens were recorded. No $2 \mathrm{~d}$-old queens dealated. The third category of queens was reproductive. We collected one or two of these queens from polygyne laboratory colonies and the sole queen heading monogyne colonies (see Table 1). The mating status of the polygyne reproductive queens was unknown [approximately one-third of such queens remain unmated in invasive populations in the USA (Ross and Keller, 1995)], whereas all monogyne reproductive queens were assumed to have mated. All reproductive queens were collected after the colonies had been maintained in the laboratory for 1 month.

All collected queens were held in glass vials at $-80^{\circ} \mathrm{C}$, for several days to several weeks, pending chemical extraction. The Gp-9 genotypes of all 14d-old queens were determined by starch gel electrophoresis following chemical extraction; $14 \mathrm{~d}$-old queens of both genotypes $(B B, B b)$ were included in the study (Table 1). Previous work has shown that reproductive queens from established polygyne colonies always possess genotype $B b$, while reproductive queens heading monogyne colonies invariably possess genotype $B B$ (Ross and Keller, 1998; Gotzek and Ross, 2007).

Chemical Extraction For piperidine analyses, queens were extracted individually in $80 \mu \mathrm{l}$ of hexane (Fluka, Buchs, Switzerland) for 2 min., and then extracted in $80 \mu \mathrm{l}$ dichloromethane (Riedel-deHaen, Seelze, Germany) for $11 \mathrm{~min}$. Adult workers were extracted as groups of 200 500 individuals in $1 \mathrm{ml}$ hexane for $2 \mathrm{~min}$., and then in $1 \mathrm{ml}$ dichloromethane for $11 \mathrm{~min}$. Solvent from the extracts was evaporated under $\mathrm{N}_{2}$, and the residue reconstituted with a known amount of hexane or dichloromethane.

We extracted individual queens and groups of workers from a second set of samples for hydrocarbon analysis. Queens were extracted in $80 \mu \mathrm{l}$ hexane for $8 \mathrm{~min}$. The solvent was evaporated under $\mathrm{N}_{2}$, and the solute was re-dissolved in $2 \mu \mathrm{l}$ hexane. Residual solvent on $14 \mathrm{~d}$-old queens was allowed to evaporate for $10 \mathrm{~min}$., after which queens were stored at $-80^{\circ} \mathrm{C}$ until determination of $G p-9$ genotype.

For hydrocarbon extraction of workers, subsets of 40 individuals were taken randomly from frozen colony samples and extracted in $100 \mu \mathrm{l}$ hexane for $8 \mathrm{~min}$. Solvent was removed from the extract by a $\mathrm{N}_{2}$ stream, and the residue re-dissolved in $20 \mu \mathrm{l}$ of hexane.

Chemical Analysis For piperidine analyses, extracts were first analyzed qualitatively on an Agilent (Santa Clara, CA, USA) 6890 gas chromatograph (GC), coupled with an Agilent 5975 mass selective (MS) detector operated in electron impact ionization mode. Peaks in extracts then were quantified by flame ionization detection (FID). GCs were operated in splitless injection mode, and were fitted with a DB-1MS column $(30 \mathrm{~m} \times 0.25 \mathrm{~mm} \times 0.25 \mu \mathrm{m}$; Agilent $)$. Column ovens were programmed from $60^{\circ}-200^{\circ} \mathrm{C}$ at $40^{\circ} \mathrm{C} \mathrm{min}^{-1}$, after an initial delay of $2 \mathrm{~min}$, then to $320^{\circ} \mathrm{C}$ at $5^{\circ} \mathrm{C} \mathrm{min}^{-1}$, and held at $320^{\circ} \mathrm{C}$ for $5 \mathrm{~min}$. The injector was set at $260^{\circ} \mathrm{C}$, the MS quad at $150^{\circ} \mathrm{C}$, MS source at $230^{\circ} \mathrm{C}$, and transfer line at $300^{\circ} \mathrm{C}$. FID temperature was set at $320^{\circ} \mathrm{C}$.

For hydrocarbon analysis, the MS was used exclusively, in order to identify some alkenes that differed with queen Gp-9 genotype.

Chemical Identification Venom alkaloids and hydrocarbons were identified based on retention indices, mass spectra, and fragmentation patterns, with these data compared with previously published data (Brand et al., 1972, 1973; Nelson et al., 1980; Chen and Fadamiro, 2009; Dall'Aglio-Holvorcem et al., 2009). Double bond positions in unsaturated alkyl chains were determined by GC-MS fragmentation patterns of dimethyl disulfide adducts, as described in Liebig et al. (2009). See Table 1 for details of diagnostic ions.

Quantitative Analysis Peaks (Table 1) were aligned across samples, with only piperidines and unsaturated hydrocarbons used in the quantitative and statistical analyses. FID peak areas from both hexane and dichloromethane extracts were added, and the percentage of each peak out of total peak area calculated.

In the second set of samples, only hydrocarbon peaks were analyzed. We detected 49 compounds that were presumed to be hydrocarbons; this number was reduced to 26 for subsequent analysis. The peak area of all 49 presumptive hydrocarbons was summed and the relative proportion of each compound calculated. Then, the mean relative peak area of each compound was calculated for all queen and worker classes. For further analysis, we selected only compounds that had a mean of at least $1 \%$ of total peak area across all classes. The total amount of these 26 most abundant compounds was used as a reference for calculation of the relative proportions of each major unsaturated alkane. Peak areas of both data sets were calculated using MSD ChemStation D.02.00.275 data analysis software (Agilent Technologies).

Statistical Analysis For piperidines, Euclidean distances were used in non-metric multi-dimensional scaling (NMDS) analyses (Kruskal, 1964), using the program PRIMER 6.0. The first two dimensions of the model always resulted in stress values below 0.05 , which is considered an excellent representation of data structure (Clarke and Warwick, 2001). The program STATISTICA 7.1 (StatSoft) was used to test for differences between monogyne and polygyne workers, and between monogyne and polygyne reproductive queens, in selected constituents 
of the chemical profiles, using the $t$-test for independent samples (these data are normally distributed; KolmogorovSmirnov test, $P>0.2$ ).

Data for ratios of $c i s-\mathrm{C}_{11} /($ cis + trans $)-\mathrm{C}_{11}$ piperidines and peak areas of alkenes/alkadienes in queens were analyzed by means of resampling procedures coupled with binomial tests; resampling procedures were necessary because the use of multiple non-reproductive and reproductive queens from many of the polygyne source colonies meant that all replicates were not independent, violating a basic assumption of most standard statistical tests. Each class of queen was compared pairwise, with respect to ratios/peak areas, to each of the other classes, in the following way. For each iteration, a single replicate of each paired queen class was randomly selected and the difference in ratios/areas recorded; only paired replicates from different colonies were accepted. A third replicate of either of the two focal classes then was randomly selected from a different source colony than used for the first two replicates, and the difference between the two same-class replicate ratios/peak areas recorded. This procedure was repeated 1000 times (with replacement), and the paired between-class and within-class differences analyzed with binomial tests. The sample sizes, used for calculating the binomial probabilities following such resampling procedures, potentially range from the number of independent source colonies (most conservative) to the number of replicates (most liberal) used in the comparison; we chose to use the number of colonies in our calculations.

\section{Results}

Differences in Venom Alkaloid Composition Among Females of Different Classes Qualitative differences were apparent in the venom alkaloid profiles of the different classes of S. invicta females (Fig. 1, Table 1). The 2d-old (sexually immature, non-reproductive) queens contained the lowest amounts of alkaloids relative to hydrocarbons, with the most abundant alkaloids being the two forms of $\mathrm{C}_{11}$ piperidine and, to a lesser extent, the trans configuration of $\mathrm{C}_{13}$ piperidines. The $14 \mathrm{~d}$-old (sexually mature, nonreproductive) queens also contained mostly $\mathrm{C}_{11}$ piperidines, but had greater proportions of both cis and trans $\mathrm{C}_{13}$ piperidines than younger queens. Reproductive queens lacked many of the trans piperidines. In contrast to queens, workers contained almost no $\mathrm{C}_{11}$ piperidines, but they had large amounts of longer-chain piperidines, especially $C_{13}$ and $\mathrm{C}_{15}$ trans forms.

NMDS analysis confirmed these differences by showing clustering of piperidine profiles of the different classes (Fig. 2). Workers were separated completely from all types of queens, and workers originating from monogyne colonies were separated from those from polygyne colonies without overlap (2D stress value: 0.02 ). With the exception of a single individual (highlighted as an outlier in Fig. 3), reproductive queens also were completely separated from all types of non-reproductive queens. There was, however, no consistent difference between the reproductive $B B$ (monogyne) queens and reproductive $B b$ (polygyne) queens. Similarly, there were no evident differences in piperidine profiles of the young non-reproductive queens of the different maturation stages and $G p-9$ genotypes.

Differences in Venom Alkaloid Composition Associated with Reproductive Status of Queens The proportion of cis piperidines $\left(\right.$ cis- $\mathrm{C}_{11} /$ totalC $\left._{11}\right)$ was not different between 2-d- and 14d-old non-reproductive queens (Figs. 1, 3A). However, reproductive queens had higher proportions of $c i s$ piperidines than did either 2d- or 14d-old queens (Fig. 3A). There also was an effect of $G p-9$ genotype on proportion of cis piperidines in 14d-old queens, with $B B$ queens having a higher proportion of $c i s-\mathrm{C}_{11}$ than $B b$ queens (Fig. 3b). In this age class, the proportion of $c i$ piperidines also was different between queens that had dealated and those that had not (Fig. 3b), in agreement with previous observations showing that $G p-9 B B$ queens dealate more readily than those with genotype $B b$ (Keller and Ross, 1999). Indeed, in the present study, there was also an association between $G p-9$ genotype and dealation, with $92.9 \%(26 / 28)$ of $B B$ queens having dealated, while only $15.7 \%$ (8/51) of $B b$ queens dealated (Fisher's exact test, $P<0.001$ ).

Differences in Venom Alkaloid Composition Between Workers from Monogyne and Polygyne Colonies Adult workers from monogyne colonies had a higher proportion of saturated piperidines than workers from polygyne colonies (Fig. 4). This difference stemmed mainly from differences in saturated/total $\mathrm{C}_{13}$ piperidine ratios.

Differences in Cuticular Hydrocarbon Composition Associated with Gp-9 Genotype and Reproductive Status of Queens All castes possessed the typical hydrocarbons found in previous studies (e.g., Cabrera et al. 2004). However, we also found additional unsaturated compounds not previously reported (Fig. 5; Table 1). The following compounds met our criterion for detailed analysis: heptacosene, nonacosene, nonacosadiene, hentriacontene, and hentriacosadiene. As for the piperidine profiles, these compounds gave profiles of reproductive queens that were distinct from those of other classes of females. In particular, these compounds were completely lacking in workers (Table 1); worker hydrocarbon profiles differed from those of all queen classes (resampling procedures coupled with binomial tests, all $P<0.05$ ). Importantly, the total relative amounts of the 

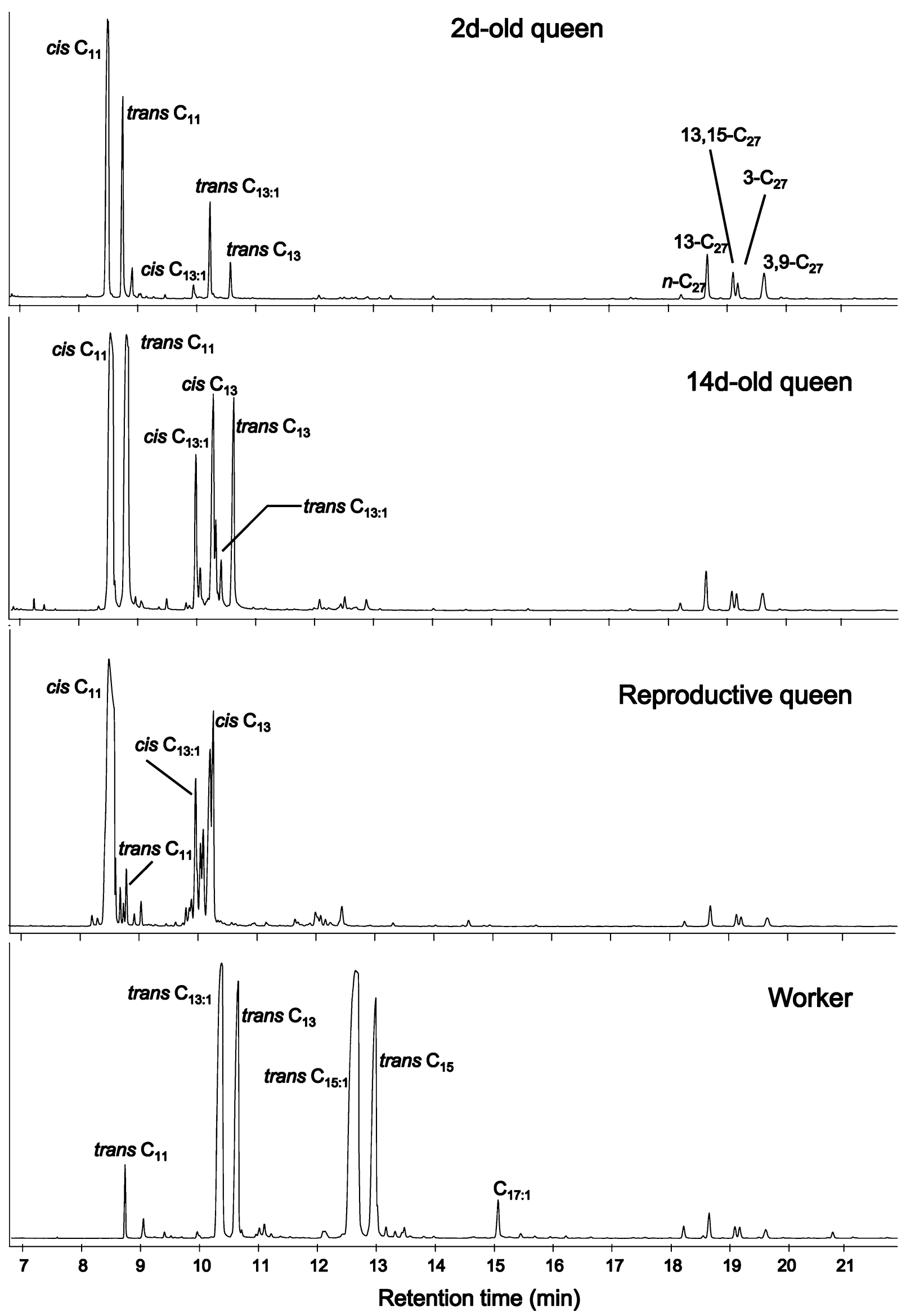
Fig. 1 Representative total ion chromatograms of cuticular extracts of different classes of queens and of a worker of Solenopsis invicta. Venom alkaloids have retention times $<17 \mathrm{~min}$, whereas cuticular hydrocarbons have retention times $>17 \mathrm{~min}$. The $2 \mathrm{~d}$-old and $14 \mathrm{~d}$-old queens originated from polygyne colonies ( $G p-9$ genotypes undetermined). The reproductive queen and worker profiles depicted are from individuals of the monogyne social form ( $G p-9$ genotype $B B$ ). See Table 1 for definitions of the chemical acronyms

focal unsaturated hydrocarbons in queens varied according to $G p-9$ genotype and reproductive status, most dramatically between reproductive queens of the alternate (social forms) genotypes (Fig. 6). Reproductive $B b$ queens from polygyne colonies had a median of $17.7 \%$ of unsaturated hydrocarbons in their profile, while reproductive $B B$ queens from monogyne colonies had a median of only $0.8 \%$ of unsaturated hydrocarbons, with one of the latter queens having no detectable amounts of unsaturated hydrocarbons.

While most (19/21) of 2d-old queens lacked any detectable amounts of unsaturated alkanes, the genotypespecific pattern of alkenes and alkadienes observed in reproductive queens had already begun to develop in queens over a 14-d period of maturation following eclosion (Fig. 6). Significantly, most 14d-old- $B b$ queens showed pronounced peaks of unsaturated hydrocarbons (median peak amount of $1.7 \%$ ), while $14 \mathrm{~d}-$ old- $B B$ queens either lacked detectable amounts or had peak amounts of $<0.1 \%$. Thus, this difference in amount of unsaturated hydrocarbons, characteristic of reproductive queens of alternate genotypes, is paralleled by smaller differences in young, non-reproductive queens of alternate genotypes.

\section{Discussion}

This study shows that both venom alkaloid and hydrocarbon profiles in the fire ant $S$. invicta are associated with caste, queen reproductive status, queen $G p-9$ genotype, and colony social organization (Electronic supplementary material Fig. 7). Venom alkaloid composition changes dramatically, both qualitatively and quantitatively, as queens become reproductively active, and also differs between workers from monogyne and polygyne colonies. Cuticular hydrocarbon profiles also show pronounced differences among different classes of queens, with the qualitative and quantitative differences observed based mainly on the amount of alkenes and alkadienes. Differences in amounts of these unsaturated hydrocarbons are related not only to queen reproductive status, but also to $G p-9$ genotype of queens, with this latter difference already noticeable at 14 days post-eclosion, when queens are sexually mature but not yet reproducing.

Previous studies showed that the venom sac of reproductive fire ant queens contains mostly $\mathrm{C}_{11}$ alkaloids, which are barely present in the sac of workers (Brand et al., 1973). We found that the proportion of cis piperidines (the less abundant geometric configuration in workers), gradually increases in the venom of queens as they become fully reproductive. Moreover, in 14d-old (mature, but nonreproductive) queens, the proportion is higher in individuals of the $G p-9 B B$ genotype than in those of the $B b$ genotype. Similarly, alkenes/alkadienes are found in significant amounts only in the queen caste, and only polygyne reproductive queens contain copious amounts. It is important to note that the difference in amounts in 14d-old queens that we detected between $B b$ and $B B$ queens is attributable to variation at the $G p-9$ gene (or variation at
Fig. 2 Results of non-metric multi-dimensional scaling analyses of piperidine venom alkaloids of different classes of Solenopsis invicta females

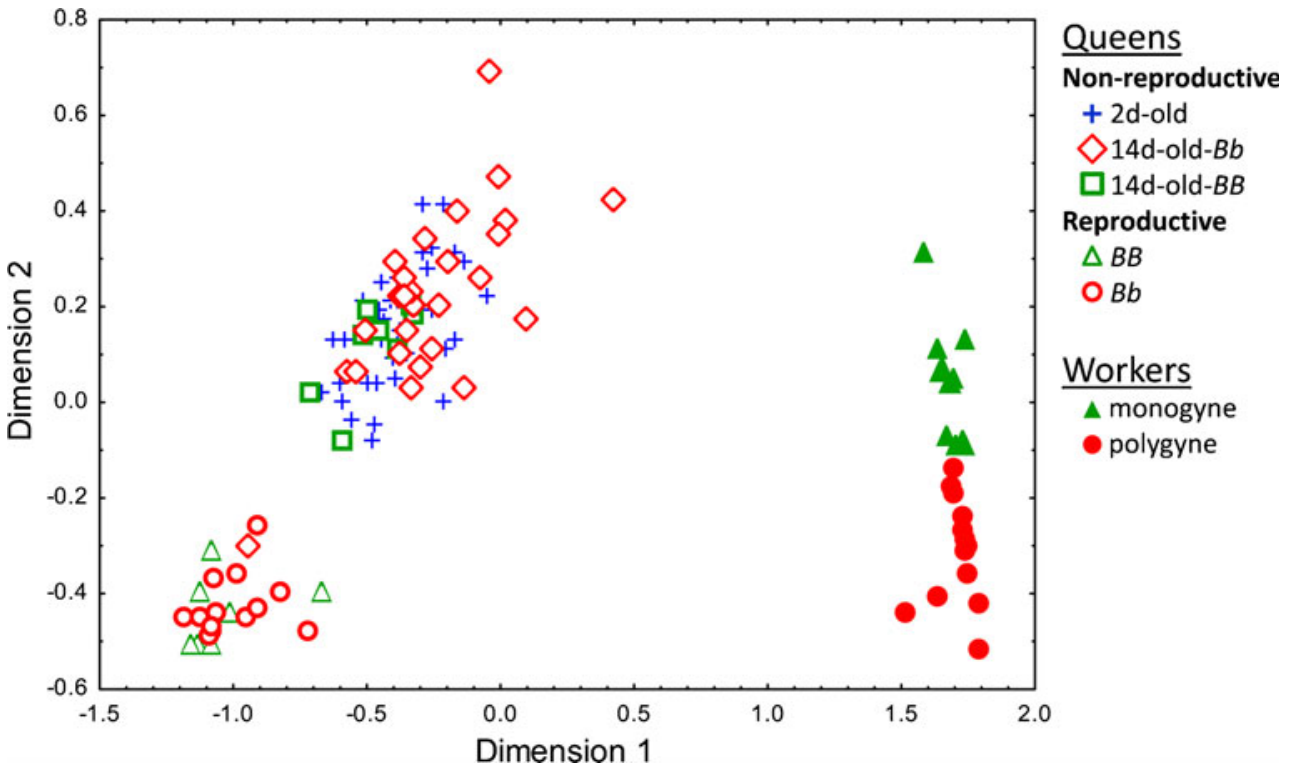




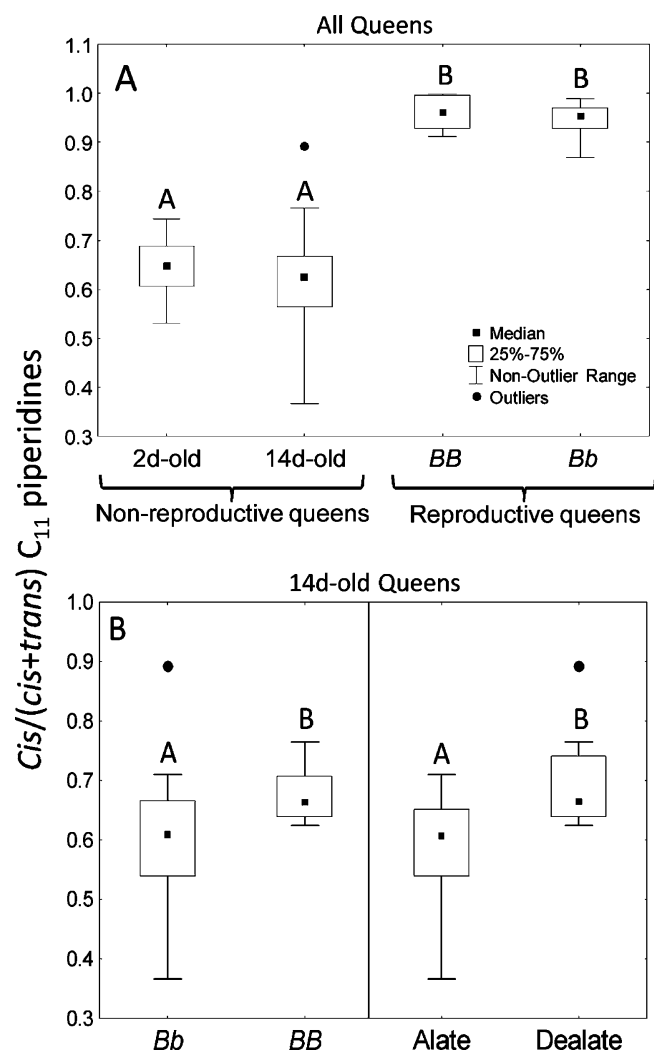

Fig. 3 Ratios of $c i s-\mathrm{C}_{11} /($ cis + trans $)-\mathrm{C}_{11}$ piperidines in different classes of Solenopsis invicta queens. Different letters within panels denote statistically significant differences. The outlier represents a dealate queen (included in the statistical analyses) evident in Fig. 2. A Patterns based on queen sexual maturity/reproductive status and on $G p-9$ genotype/social form of origin (resampling procedures coupled with binomial tests: $2 \mathrm{~d}$-old queens vs. $B B$ or $B b$ reproductive queens, both $P<0.001 ; 14 \mathrm{~d}$-old queens vs. $B B$ or $B b$ reproductive queens, both $P<0.001$; 2d-old vs. $14 \mathrm{~d}$-old queens, $P=0.17$; $t$-test for independent samples: $B B$ vs. $B b$ reproductive queens, $P=0.42$ ). B Patterns based on $G p-9$ genotype and dealation status of $14 \mathrm{~d}$-old queens (resampling procedures coupled with binomial tests: genotype $B b$ vs. $B B, P<$ 0.001 ; alates vs. dealates, $P<0.001)$. $C i s-\mathrm{C}_{11}=c i s$-2-methyl-6- $n$-undecylpiperidine; trans- $\mathrm{C}_{11}=$ trans-2-methyl-6- $n$-undecylpiperidine

linked genes), rather than to effects of social environment, because all 14d-old queens in our study originated from polygyne source colonies. This initial difference, attributable to genotype, becomes more pronounced as queens age, accelerate oogenesis, and become fully competent reproductives.

That profiles of cis piperidines and unsaturated hydrocarbons differ between sexually mature $B B$ and $B b$ queens suggests that these suites of chemicals constitute signals that workers use to recognize and discriminate between queens of the two genotypes. Importantly, such behavioral discrimination by workers only becomes apparent once queens become sexually mature (Ross and Keller, 1998; Gotzek and Ross, 2007), suggesting an association between ontogeny of these presumptive queen chemical signals and discrimination behavior directed toward them.
Previous studies showed that $14 \mathrm{~d}$-old- $B B$ queens accumulate greater energy reserves, dealate more readily, and lay more eggs immediately upon dealation than $14 \mathrm{~d}$-old- $B b$ queens; i.e., sexually mature $B B$ queens are at a more advanced state of reproductive development (Keller and Ross, 1993b; Ross and Keller, 1998; DeHeer, 2002). Thus, changes in alkaloid and hydrocarbon compositions are closely tied to physiological changes that accompany sexual maturation and the onset of reproduction that, in turn, are related to $G p-9$ genotype. Specifically, proportions of $c i s-\mathrm{C}_{11}$ piperidines increase more rapidly during maturation in $B B$ than $B b$ queens before attaining high proportions in reproductives of both genotypes, while amounts of alkenes/ alkadienes increase more rapidly in maturing $B b$ than $B B$ queens before reaching extremely high levels in $B b$ reproductives only. In line with this, $14 \mathrm{~d}$-old queens that dealated (i.e., those in a more advanced reproductive state, most of which lacked allele $b$ ) also had a higher proportion of cis piperidines than queens of the same age that had not dealated (most of which possessed allele $b$ ). Our results support tenets of the model of regulation of queen number proposed by Gotzek and Ross (2007), which invokes two distinct signaling components, one (piperidines) communicating actual or potential fertility status, and the other (alkenes/alkadienes) communicating $G p-9$ genotype. We note, however, that our data are not fully congruent with the model, which predicts that workers bearing the $b$ allele should display the same chemical profile as queens bearing this allele; workers lacked the unsaturated hydrocarbons that were differentially abundant in queens of alternate $G p-9$ genotypes. There are several possible explanations for this incongruence, including the two classes of chemicals acting synergistically, rather than as distinct components, hydrocar-

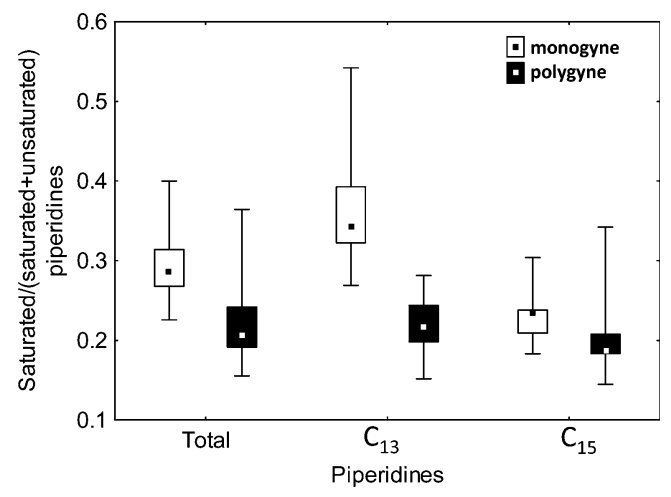

Fig. 4 Ratios of saturated to total piperidines in Solenopsis invicta workers from monogyne ( $G p-9$ genotype $B B$ only) and polygyne (mixed Gp-9 genotypes) colonies. Comparisons of total piperidines, $\mathrm{C}_{13}$ piperidines, and $\mathrm{C}_{15}$ piperidines, respectively, are shown. Only total and $\mathrm{C}_{13}$ piperidines are different in ratios $(t$-test for independent samples: total, $t=3.46, P<0.003 ; \mathrm{C}_{13}, t=6.05, P<0.001 ; \mathrm{C}_{15}, t=1.72$, $P=0.098$ ) 
Fig. 5 Representative cuticular hydrocarbon profiles of Solenopsis invicta reproductive queens of $G p-9 B b$ (polygyne queen) and $B B$ (monogyne queen) genotypes. The highlighted partial profile of the $B B$ queen is at the same scale as the profile of the $B b$ queen.

Numbers correspond to compounds listed in Table 1. Any hydrocarbons eluting before $15 \mathrm{~min}$ are not shown. Asterisk indicates a contaminant

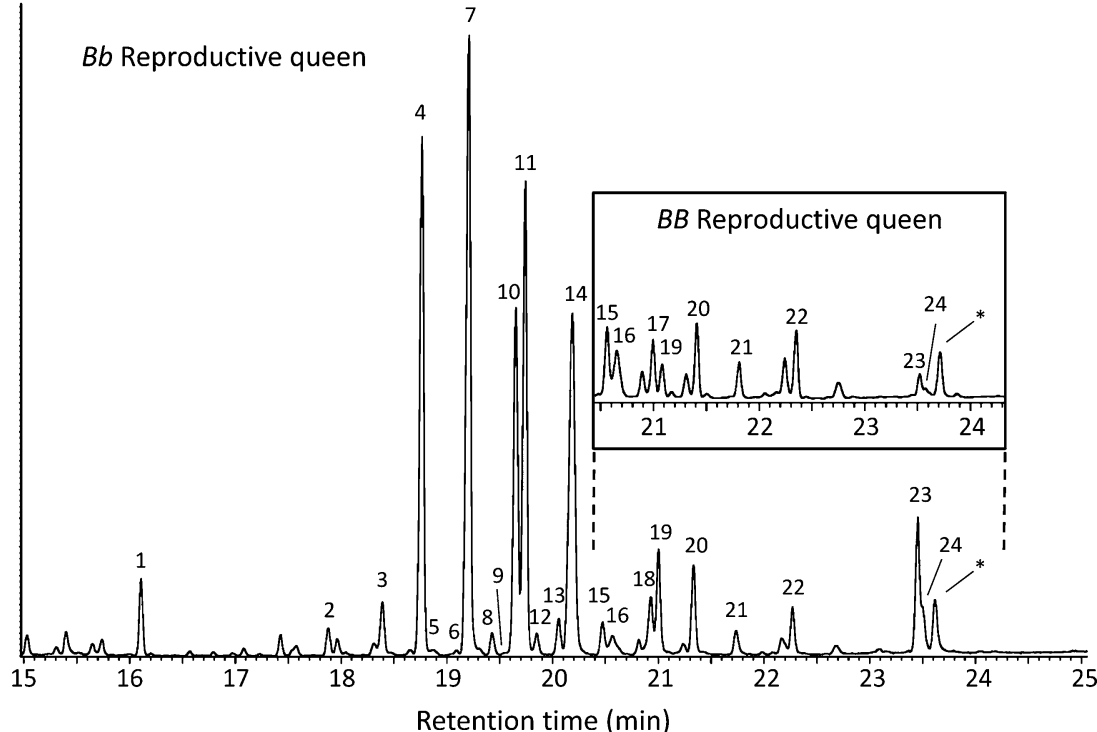

bon expression depending jointly on genotype and fertility status, or other classes of chemicals on the cuticle surface, including perhaps the GP-9 protein itself (unpublished data), being involved in signaling $G p-9$ genotype in $S$. invicta.

To date, fertility signaling has been assigned to cuticular and egg surface hydrocarbons in most social insects in which this effect has been investigated (Monnin, 2006; Le Conte and Hefetz, 2008; Peeters and Liebig, 2009; Liebig, 2010). In $S$. invicta, it appears that both hydrocarbons and piperidines may play roles in providing information relevant to regulation of colony queen number, as described above. Like hydrocarbons, piperidines are derived from acetate (Leclercq et al., 1996), raising the possibility that

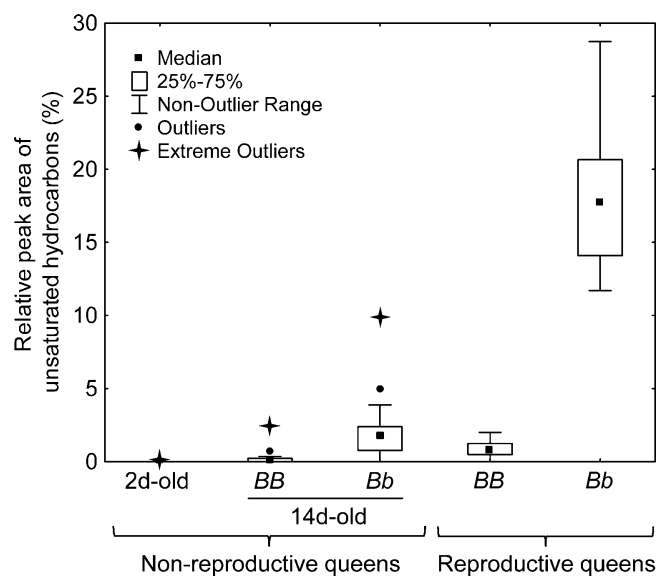

Fig. 6 Alkene and alkadiene relative amounts in different classes of Solenopsis invicta queens. All pairwise differences are significant (resampling procedures coupled with binomial tests: 2 d-old queens vs. $14 \mathrm{~d}-$ old- $B B$ queens, and $14 \mathrm{~d}$-old- $B b$ queens vs. $B B$ reproductive queens, both $P<0.05 ; 14 \mathrm{~d}$-old- $B b$ queens vs. $2 \mathrm{~d}$-old and $14 \mathrm{~d}$-old- $B B$ queens, both $P<0.01$; all other combinations, $P<0.001$ ) similar metabolic processes regulate the synthesis of hydrocarbon chains and piperidines.

Previously, piperidines were found not to be attractive in S. invicta (Vander Meer et al., 1980). The attractiveness of the queen poison gland secretion is attributed to two pyranones identified by Rocca et al. (1983a,b). Nevertheless, these pyranones do not delay the onset of reproduction in young queens, a primer effect that the poison sac was found to produce (Fletcher and Blum, 1981; Glancey et al., 1984; Vargo, 1998). Piperidines, with their large amounts dominating poison sac content, could be potential players in this phenomenon.

The ratio of saturated piperidines in adult worker cuticular extracts was higher in S. invicta workers from monogyne colonies than in workers from polygyne colonies (Fig. 4). This is in agreement with previous findings of differences in alkaloid composition between workers of the two social forms (Lai et al., 2008; Lin et al., 2010). Quantitative differences in piperidine composition have also been found between minor and major workers, as well as among workers of different ages (Deslippe and Guo, 2000), suggesting that piperidines also may be used for caste and possibly nestmate recognition.

Many ants use secretions from the metapleural gland as antiseptics for their nest and body surface. Fire ants have fatty acids with antimicrobial properties in the metapleural glands that may be used for that purpose (Cabrera et al., 2004), although venom alkaloids also may take part in defense against microbial pathogens. Workers of $S$. invicta display gaster-flagging behavior, whereby they spray the contents of the poison gland onto the nest and their body (Obin and Vander-Meer, 1985). Indeed, venom alkaloids dominate in solid phase microextraction (SPME) extracts of Solenopsis xyloni, even when the gaster cuticle is excluded 
from extraction (unpublished data). As high molecular weight compounds, these alkaloids are nonvolatile (MacConnell et al., 1971) and potentially can remain on the cuticle for long periods. Considering the above, it is not surprising that piperidines also are found in abundance throughout the nest (Chen, 2007). The large quantities of venom alkaloids produced for a possible role in protecting ants from microbial pathogens may have facilitated their co-option to serve as fertility signals within colonies, especially if a queen's venom becomes distributed on her cuticle. Thus, the present study suggests that venom alkaloids of fire ants may have secondarily evolved roles in communication that typically are assigned to cuticular hydrocarbons in many other social insects (Monnin, 2006; Le Conte and Hefetz, 2008; Peeters and Liebig, 2009; Liebig, 2010). Despite the large amounts of alkaloids, hydrocarbons still may play a role in regulating social structure by signaling queen $G p-9$ genotype. Future manipulative behavioral studies should directly examine whether, and if so how, these and other compounds found on the queen cuticle, including GP-9 protein, function in maintaining the structure of fire ant societies.

Acknowledgements The study was partly funded by Swiss National Science Foundation grants to LK and by the Center for Insect Science through NIH Training Grant \# 1K12 GM000708 to DE.

\section{References}

ANDERSON, J. and VANDER-MEER, R. 2001. Experimental evidence against cuticular hydrocarbons as nestmate recognition cues in the fire ant, Solenopsis invicta. Proceedings of the 2001 Annual Imported Fire Ant Research Conference, San Antonio, TX, February 28-March 2, 2001:69-70.

Brand, J. M., Blum, M. S., FAles, H. M., and Macconnell, J. G. 1972. Fire ant venoms - comparative analyses of alkaloidal components. Toxicon 10:259-271

Brand, J. M., Blum, M. S., and Ross, H. H. 1973. Biochemical evolution in fire ant venoms. Insect Biochem. 3:45-51.

Brent, C. S. and VARGO, E. L. 2003. Changes in juvenile hormone biosynthetic rate and whole body content in maturing virgin queens of Solenopsis invicta. J. Insect Physiol. 49:967-974.

Cabrera, A., Williams, D., Hernandez, J., Caetano, F., and JAFFE, K. 2004. Metapleural- and postpharyngeal-gland secretions from workers of the ants Solenepsis invicta and S. geminata. Chem. Biodivers. 1:303-311

CHEN, J. 2007. Qualitative analysis of red imported fire ant nests constructed in silica gel. J. Chem. Ecol. 33:631-642.

Chen, L. and Fadamiro, H. Y. 2009. Re-investigation of venom chemistry of Solenopsis fire ants. II. Identification of novel alkaloids in S. invicta. Toxicon 53:479-486.

Clarke, K. R. and WARWICK R. M. 2001. Change in Marine Communities: An Approach to Statistical Analysis and Interpretation. $2^{\text {nd }}$ Edition, Primer-E, Plymouth

Dall'aglio-Holvorcem, C. G., Benson, W. W., Gilbert, L. E., Trager, J. C., and Trigo, J. R. 2009. Chemical tools to distinguish the fire ant species Solenopsis invicta and S. saevissima (Formicidae: Myrmicinae) in Southeast Brazil. Biochem. Syst. Ecol. 37:442-451.
DEHEER, C. J. 2002. A comparison of the colony-founding potential of queens from single- and multiple-queen colonies of the fire ant Solenopsis invicta. Anim. Behav. 64:655-661.

Deheer, C. J., Goodisman M. A. D., and Ross. K. G. 1999. Queen dispersal strategies in the multiple-queen form of the fire ant Solenopsis invicta. Am. Nat. 153:660-675.

DESLIPPE, R. and GuO, Y. 2000. Venom alkaloids of fire ants in relation to worker size and age. Toxicon 38:223-232.

D'ettorre, P., Heinze, J., Schulz, C., Francke, W., and Ayasse, M. 2004. Does she smell like a queen? Chemoreception of a cuticular hydrocarbon signal in the ant Pachycondyla inversa. $J$. Exp. Biol. 207:1085-1091.

Endler, A., Liebig, J., Schmitt, T., PArker, J. E., Jones, G. R., SCHREIER, P., and Hölldobler, B. 2004. Surface hydrocarbons of queen eggs regulate worker reproduction in a social insect. Proc. Natl. Acad. Sci. USA 101:2945-2950.

Endler, A., Liebig, J., and Hölldobler, B. 2006. Queen fertility, egg marking and colony size in the ant Camponotus floridanus. Behav. Ecol. Sociobiol. 59:490-499.

Fletcher, D. J. C. and Blum, M. S. 1981. Pheromonal control of dealation and oogenesis in virgin queen fire ants. Science 212:73-75.

Glancey, B., RoccA, J., Lofgren, C., and Tumlinson, J. 1984. Field-tests with synthetic components of the queen recognition pheromone of the red imported fire ant, Solenopsis invicta. Sociobiology 9:19-30.

GotZeK, D. and Ross, K. G. 2007. Genetic regulation of colony social organization in fire ants: an integrative overview. Quart. Rev. Biol. 82:201-226.

Hannonen, M., Sledge, M., Turillazzi, S., and Sundström, L. 2002. Queen reproduction, chemical signalling and worker behaviour in polygyne colonies of the ant Formica fusca. Anim. Behav. 64:477-485.

Holman, L., Jørgensen, C. G., Nielsen, J., and D’ettore, P. 2010. Identification of an ant queen pheromone regulating worker sterility. Proc. R. Soc. B. 277:3793-3800.

Keller, L. and NonaCS, P. 1993. The role of queen pheromones in social insects-queen control or queen signal? Anim. Behav. 45:787-794.

Keller, L. and Ross, K. G. 1993a. Phenotypic plasticity and "cultural" transmission of alternative social organizations in the fire ant Solenopsis invicta. Behav. Ecol. Sociobiol. 33:121-129.

Keller, L. and Ross, K. G. 1993b. Phenotypic basis of reproductive success in a social insect: genetic and social determinants. Science 260:1107-1110.

Keller, L. and Ross, K. G. 1998. Selfish genes: a green beard in the red fire ant. Nature 394:573-575.

KelleR, L. and Ross, K. G. 1999. Major gene effects on phenotype and fitness: the relative roles of Pgm-3 and $G p-9$ in introduced populations of the fire ant Solenopsis invicta. J. Evol. Biol. 12:672-680.

KRUSKAL, J. B. 1964. Multidimensional scaling by optimizing goodness of fit to a nonmetric hypothesis. Psychometrika 29:1-27.

LAI, L., HuANG, R., and WU, W. 2008. Venom alkaloids of monogyne and polygyne forms of the red imported fire ant, Solenopsis invicta, in Taiwan. Insectes Sociaux 55:443-449.

LeclercQ, S., Braekman, J., Daloze, D., Pasteels, J., and VANDER-MEER, R. 1996. Biosynthesis of the solenopsins, venom alkaloids of the fire ants. Naturwissenschaften 83:222-225.

Le Conte, Y. and Hefetz, A. 2008. Primer pheromones in social Hymenoptera. Annu. Rev. Entomol. 53:523-542.

LIEBIG, J. 2010. Hydrocarbon profiles indicate fertility and dominance status in ant, bee, and wasp colonies, pp 254-260, in G. J. Blomquist and A. G. Bagnères (eds.), Insect Hydrocarbons: Biology, Biochemistry, and Chemical Ecology. Cambridge University Press, Cambridge. 
Liebig, J., Eliyahu, D., and Brent, C. S. 2009. Cuticular hydrocarbon profiles indicate reproductive status in the termite Zootermopsis nevadensis. Behav. Ecol. Sociobiol. doi:10.1007/ s00265-009-0807-5.

LIN, Y.-K., ChANG, H.-Y., Wu, W.-J., Ho, H.-Y., and LIN, C.-C. 2010. Different cuticular chemical profiles between the monogynous and polygynous forms of the red imported fire ant, Solenopsis invicta (Hymenoptera: Formicidae) in Taiwan. Sociobiology $56: 39-55$

Macconnell, J. G., Blum, M. S., and Fales, H. M. 1971. The chemistry of fire ant venom. Tetrahedron 26:1129-1139.

Monnin, T. 2006. Chemical recognition of reproductive status in social insects. Ann. Zool. Fenn. 43:515-530.

Nelson, D. R., Fatland, C. L., Howard, R. W., McDaniel, C. A., and BlomQuist, G. J. 1980. Re-analysis of the cuticular methylalkanes of Solenopsis invicta and Solenopsis richteri. Insect Biochem. 10:409-418.

OBIn, M. S. and VANDER-MeER, R. K. 1985. Gaster flagging by fire ants (Solenopsis spp.): Functional significance of venom dispersal behavior. J. Chem. Ecol. 11:1757-1767.

ORTIUS, D. and HeINZE, J. 1999. Fertility signaling in queens of a North American ant. Behav. Ecol. Sociobiol. 45:151-159.

Peeters, C. and LieBig, J. 2009. Fertility signaling as a general mechanism of regulating reproductive division of labor in ants, pp 220-242, in: J. Gadau and J. Fewell (eds.), Organization of Insect Societies: From Genome to Socio-Complexity. Harvard University Press.

Rocca, J. R., Tumlinson, J. H., Glancey, B. M., and Lofgren, C. S. 1983a. Synthesis and stereochemistry of tetrahydro-3, 5dimethyl-6-(1)methylbutyl)-2H-pyran-2-one, a component of the queen recognition pheromone of Solenopsis invicta. Tetrahedron Lett. 24:1893-1896.

Rocca, J. R., Tumlinson, J. H., Glancey, B. M., and Lofgren, C. S. 1983b. The queen recognition pheromone of Solenopsis invicta, preparation of (-6-(1-pentenyl)-2H-pyran-2-one. Tetrahedron Lett. 24:1889-1892.

Ross, K. G. 1988. Differential reproduction in multiple-queen colonies of the fire ant Solenopsis invicta (Hymenoptera: Formicidae). Behav. Ecol. Sociobiol. 23:341-355.
Ross, K. G. and Keller, L. 1995. Ecology and evolution of social organization: Insights from fire ants and other highly eusocial insects. Annu. Rev. Ecol. Syst. 26:631-656.

Ross, K. G. and KELLER, L. 1998. Genetic control of social organization in an ant. Proc. Natl. Acad. Sci., USA 95:14232-14237.

Ross, K. G. and KeLLER, L. 2002. Experimental conversion of colony social organization by manipulation of worker genotype composition in fire ants (Solenopsis invicta). Behav. Ecol. Sociobiol. 51:287-295.

Smith, A. A., Hölldobler, B., and Liebig, J. 2008. Hydrocarbon signals explain the pattern of worker and egg policing in the ant Aphaenogaster cockerelli. J. Chem. Ecol. 34:1275-1282.

Tschinkel, W. 2006. The Fire Ants. Harvard University Press, Cambridge, MA.

VAnder-Meer, R. and Morel, L. 1995. Ant queens deposit pheromones and antimicrobial agents on eggs. Naturwissenschaften 82:93-95.

Vander Meer, R. K., Glancey, B. M., Lofgren, C. S., Glover, A., Tumlinson, J. H., and RocCA, J. 1980. The poison sac of red imported fire ant queens: Source of a pheromone attractant. Ann. Entomol. Soc. Am. 73:609-612.

VAnder Meer, R. K., Glancey, B. M., and Lofgren, C. S. 1982. Biochemical changes in the crop, esophagus and postpharyngeal gland of colony-founding red imported fire ant queens (Solenopsis invicta). Insect Biochem. 12:123-127.

VAN ZWEDEN, J. S., and D'ETTORRE, P. 2010. Nestmate recognition in social insects and the role of hydrocarbons, pp 222-243, in: G. J. Blomquist and A. G. Bagnères (eds.), Insect hydrocarbons: Biology, biochemistry, and chemical ecology. Cambridge University Press, Cambridge, UK

VARGO, E. 1998. Primer pheromones in ants, pp 293-313, in: M. D. Breed, K. E. Espelie, R. K. Vander Meer and M. L. Winston (eds.), Pheromone Communication in Social Insects: Ants, Wasps, Bees, and Termites. Westview Press, Boulder, CO.

VARGO, E. and HulSEY, C. 2000. Multiple glandular origins of queen pheromones in the fire ant Solenopsis invicta. J. Insect Physiol. 46:1151-1159.

Weil, T., Hoffmann, K., Kroiss, J., Strohm, E., and Korb, J. 2009. Scent of a queen - cuticular hydrocarbons specific for female reproductives in lower termites. Naturwissenschaften 96: 315-319. 